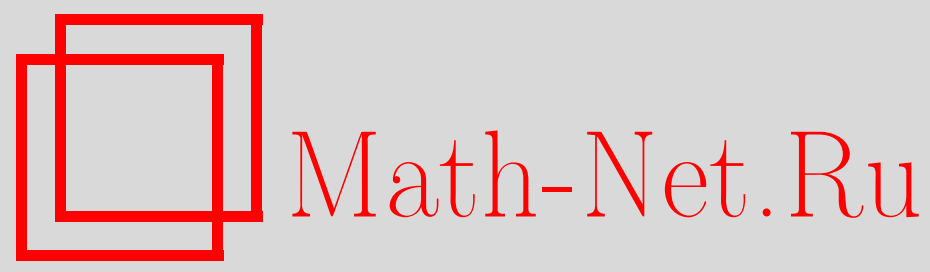

Т. Г. Квирикашвили, А. А. Лашхи, Перспективные отображения модулей, Матем. заметки, 2005, том 77, выпуск 6, 946-947

DOI: https://doi.org/10.4213/mzm2551

Использование Общероссийского математического портала Math-Net.Ru подразумевает, что вы прочитали и согласны с пользовательским соглашением http://www.mathnet.ru/rus/agreement

Параметры загрузки:

IP : 52.205 .19 .152

26 апреля 2023 г., $11: 30: 00$

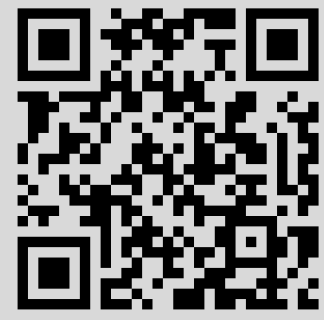




\section{ПЕРСПЕКТИВНЫЕ ОТОБРАЖЕНИЯ МОДУЛЕЙ}

\section{Т.Г. Квирикашвили, А.А. Лашхи}

Одной из основных проблем геометрической алгебры является представление специфических с геометрической точки зрения отображений (перспективы, коллинеации, гармонические отображения и др.) через (полу) линейные функции. Такого рода теоремы назьпаются основными теоремами геометрической алгебры [1]-[3].

Р. Бэрн, Дж. фон Нейманн, Ж. Дьедонне предложили идею расширения теории для общих основных колец, тем самым дав стимул активности в этом направлении [1]-[3]. Однако заметим, что ни одна кольевая версия о перспективных отображениях не известна. Причина в том, что в отличии от классического случая не каждый подмодуль свободного $R$-модуля имеет прямое дополнение.

Кольцо $R$ назовем $S F$-жольиом, если любой подмодуль свободного $R$-модуля $X$ свободен и $X$ обладает инвариантным базисным числом. Пусть $\Omega$ - свободный модуль над $S F$-кольцом $R$, $\operatorname{dim} \Omega=N \geqslant 3, N<\infty$. Пусть $M$ и $M^{\prime}$ - подмодули одинакового ранга $n, 2 \leqslant n<N$.

ОПРЕДЕЛЕНИЕ 1 . Подмодуль $T \subset \Omega$ называется $\Delta$-дополнением к подмодулю $M$, если

$$
M \cap T=0, \quad M \oplus T \cong \Omega
$$

ПрЕДЛОЖЕнИЕ 1. Подмодули $M$ и $M^{\prime}$ имеют общее $\Delta$-дополнение $T$, m.e.

$$
M \cap T=M \cap T^{\prime}=0, \quad M \oplus T \cong M^{\prime} \oplus T \cong \Omega .
$$

ПРЕДЛОЖЕНИЕ 2. Имеют место следующие соотношения:

(I) $(M \oplus T) \cap\left(M^{\prime} \oplus T\right)=\Omega_{1} \cong \Omega$;

(II) ecлu $M_{1}=M \cap \Omega_{1}, M_{1}^{\prime}=M^{\prime} \cap \Omega_{1}$, mo $M_{1} \oplus T=M_{1}^{\prime} \oplus T=\Omega_{1}$;

(III) eсли $U_{1} \subset M_{1}, W \subset \Omega_{1}, T \subset W$, mo $U_{1} \subset M_{1} \cap W \Longleftrightarrow W=U_{1} \oplus T$.

Пусть $U_{1} \subset M_{1}$-произвольный подмодуль и $W=U_{1} \oplus T$. Рассмотрим пересечение $W \cap M^{\prime}=$ $U_{1}^{\prime}$. Имеем $W=U_{1}^{\prime} \oplus T$. Тогда между подмодулями $U_{1} \subset M_{1}$ и $U_{1}^{\prime} \subset M_{1}^{\prime}$ устанавливается взаимно однозначное соответствие, определенное равенством

$$
U_{1} \oplus T=U_{1}^{\prime} \oplus T
$$

Пусть $\mathbb{P}\left(M_{1}\right)$ и $\mathbb{P}\left(M_{1}^{\prime}\right)$ - проективные пространства; напомним, что проективное пространство $\mathbb{P}\left(M_{1}\right)$ - это множество всех свободных прямых слагаемых ранга 1 , т.е.

$$
\mathbb{P}\left(M_{1}\right)=\left\{R e, \text { где } e \in M_{1} \text { - унимодулярный элемент }\right\} .
$$

Элемент $e \in M_{1}$ унимодулярен, если существует линейная форма $g: M_{1} \rightarrow R$ такая, что $g(e)=1$.

ОПРЕДЕЛЕНИЕ 2 . Биекцию $p: \mathbb{P}\left(M_{1}\right) \rightarrow \mathbb{P}\left(M_{1}^{\prime}\right)$ назовем перспективой с иентром перспективы $\mathbb{P}(T)$, если она определяется следуюшим равенством:

$$
R m \oplus T=R m^{\prime} \oplus T, \quad R m \in \mathbb{P}\left(M_{1}\right), \quad R m^{\prime} \in \mathbb{P}\left(M^{\prime}\right) .
$$

В дальнейшем все модули определены над $S F$-кольцом.

Теорема 1. Перспектива $p: \mathbb{P}\left(M_{1}\right) \rightarrow \mathbb{P}\left(M_{1}^{\prime}\right)$ индучируется линейньм отображсением $\alpha: M_{1} \rightarrow M_{1}^{\prime}$, тождественным на пересечении $M_{1} \cap M_{1}^{\prime}$. 
ОПРЕДЕЛЕниЕ 3 . Биекцию $\Delta p: \mathbb{P}(M) \rightarrow \mathbb{P}\left(M^{\prime}\right)$ назовем $\Delta$-перспективой с центром $\mathbb{P}(T)$, если найдутся подмодули $M_{1} \subset M$ и $M_{1}^{\prime} \subset M^{\prime}$ такие, что между $\mathbb{P}\left(M_{1}\right)$ и $\mathbb{P}\left(M_{1}^{\prime}\right)$ можно установить перспективу $p$ с центром $\mathbb{P}(T)$ и следующая диаграмма коммутативна:

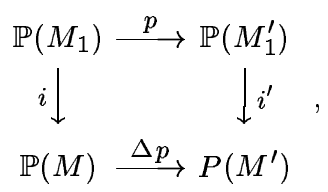

т.e. $\Delta p \circ i=i^{\prime} \circ p$

$$
\begin{aligned}
& i: R m \rightarrow R e, \quad R m=R e \cap M_{1}, \quad R m \in \mathbb{P}\left(M_{1}\right), \quad R e \in \mathbb{P}(M) ; \\
& i^{\prime}: R m^{\prime} \rightarrow R e^{\prime}, \quad R m=R e^{\prime} \cap M_{1}^{\prime}, \quad R m^{\prime} \in \mathbb{P}\left(M_{1}^{\prime}\right), \quad R e^{\prime} \in \mathbb{P}\left(M^{\prime}\right) \text {. }
\end{aligned}
$$

Tеорема $1^{\prime}$. $\Delta$-перспектива $\Delta p: \mathbb{P}(M) \rightarrow \mathbb{P}\left(M^{\prime}\right)$ индуцируется линейным отображением $\alpha: M_{1} \rightarrow M_{1}^{\prime}$, тохдественным на әлементах пересечения $M \cap M^{\prime}=M_{1} \cap M_{1}^{\prime}$.

Лемма. Линейное отображение $\alpha: M_{1} \rightarrow M_{1}^{\prime}$ тождественно на әлементах пересечения $M_{1} \cap M_{1}^{\prime}$ тогда и только тогда, когда

$$
M_{1}=\left(M_{1} \cap M_{1}^{\prime}\right) \oplus M_{0}, \quad M_{1}^{\prime}=\left(M_{1} \cap M_{1}^{\prime}\right) \oplus M_{0}^{\prime} .
$$

Биекцию $\beta: \mathbb{P}\left(M_{1}\right) \rightarrow \mathbb{P}\left(M_{1}^{\prime}\right)$ назовем колине ацией, если

$$
R e_{1} \subset R e_{2}+R e_{3} \Longleftrightarrow \beta\left(R e_{1}\right) \subset \beta\left(R e_{2}\right)+\beta\left(R e_{3}\right) ;
$$

биекцию назовем проекцией, если ее можно получить в результате умножения колинеации.

Теорема 2. Колинеация $\beta: \mathbb{P}\left(M_{1}\right) \rightarrow \mathbb{P}\left(M_{1}^{\prime}\right)$ является перспективой тогда и только тогда, когда она индуцируется линейным отображением модулей $M_{1}$ и $M_{1}^{\prime}$, тождественным на пересечении $M_{1} \cap M_{1}^{\prime}$.

Теорема 3. Пусть $\beta: \mathbb{P}\left(M_{1}\right) \rightarrow \mathbb{P}\left(M_{1}^{\prime}\right)$ - колинеачия, тождественная на пересечении

$$
\mathbb{P}\left(M_{1}\right) \cap \mathbb{P}\left(M_{1}^{\prime}\right)=\mathbb{P}\left(M_{1} \cap M_{1}^{\prime}\right) .
$$

Тогда

(i) если $\operatorname{dim}\left(M_{1} \cap M_{1}^{\prime}\right) \geqslant 2$, то $\beta$ - перспектива;

(ii) если $M_{1} \cap M_{1}^{\prime}=0$ и $\beta$ - проекчия, то $\beta$ - перспектива;

(iii) если $\operatorname{dim}\left(M_{1} \cap M_{1}^{\prime}\right)=1, \beta-$ проекиия и $R$ коммутативно, то $\beta$ - перспектива.

ЗАмечАниЕ. Если $R$ - некоммутативное кольцо, то можно построить соответствующий пример, опровергающий (iii).

\section{СПИСОК ЦИТИРОВАННОЙ ЛИТЕРАТУРЫ}

1. F. Buekenhout (ed. ) Handbook of Incidence Geometry. Amsterdam: North Holland, 1995. 2. Bartolone C., Bartolozzi F. // Rings and Geometry / ed. R. Kaya, P. Plaumann, K. Strambach. NATO ASI Series: D. Reidel Publ., 1985. P. 353-389. 3. McDonald B. Linear Algebra over Commutative Rings. New York: Marcel Dekker, 1984. 